\title{
Myosin IIB Is Required for Growth Cone Motility
}

\author{
Paul C. Bridgman, ${ }^{1}$ Sonya Dave, ${ }^{1}$ Clara F. Asnes, ${ }^{2}$ Antonella N. Tullio, ${ }^{3}$ and Robert S. Adelstein ${ }^{3}$ \\ Departments of ${ }^{1}$ Anatomy and Neurobiology and ${ }^{2}$ Biochemistry and Molecular Biophysics, Washington University School \\ of Medicine, St. Louis, Missouri 63110, and ' ${ }^{2}$ aboratory of Molecular Cardiology, National Heart, Lung, and Blood \\ Institute, National Institutes of Health, Bethesda, Maryland 20892
}

Growth cones are required for the forward advancement and navigation of growing axons. Modulation of growth cone shape and reorientation of the neurite are responsible for the change of outgrowth direction that underlies navigation. Change of shape involves the reordering of the cytoskeleton. Reorientation of the neurite requires the generation of tension, which is supplied by the ability of the growth cone to crawl on a substrate. The specific molecular mechanisms responsible for these activities are unknown but are thought to involve actomyosin-generated force combined with linkage to the cell surface receptors that are responsible for adhesion (Heidemann and Buxbaum, 1998). To test whether myosin IIB is responsible for the force generation, we quantified shape dynamics and filopodial-mediated traction force in growth cones from myosin IIB knock-out (KO) mice and compared them with neurons from normal littermates. Growth cones from the KO mice spread less, showed alterations in shape dynamics and actin organization, and had reduced filopodial-mediated traction force. Although peak traction forces produced by filopodia of $\mathrm{KO}$ cones were decreased significantly, KO filopodia occasionally developed forces equivalent to those in the wild type. This indicates that other myosins participate in filopodial-dependent traction force. Therefore, myosin IIB is necessary for normal growth cone spreading and the modulation of shape and traction force but acts in combination with other myosins for some or all of these activities. These activities are essential for growth cone forward advancement, which is necessary for outgrowth. Thus outgrowth is slowed, but not eliminated, in neurons from the myosin IIB KO mice.

Key words: growth cones; motility; myosin; actin; traction force; knock-out mice
Two components of growth cone motility appear to be necessary for directing axons to their targets. One is the formation of actin-rich filopodia and lamellipodia in the direction of growth. Filopodia are essential for directed outgrowth (Bentley and Toroian-Raymond, 1986; Chien et al., 1993). They are necessary for turning in response to environmental cues (Zheng et al., 1996) and are a unique sensory motor apparatus (Kater and Rehder, 1995). Lamellipodia also contribute to shape changes during turning. Actin polymerization drives the formation of filopodia and lamellipodia (Mallavarapu and Mitchison, 1999), but other factors contribute to the modulation of the size, rate, shape, and site of formation. Myosins have been implicated as modulators.

Another component of growth cone motility is the exertion of force on the substratum (Heidemann et al., 1990). Growth cones produce traction force and pull on neurites during outgrowth (Lamoureux et al., 1989). This is required for normal advancement. Tension reorients the neurite when the growth cone crawls along a new trajectory. The mechanism of traction force generation remains controversial. Recently, a model has been presented that integrates myosin II-dependent contractile (Heidemann et al., 1990) and flow-driven (Lin et al., 1996) mechanisms as the basis for fish keratocyte movement (Svitkina et al., 1997). Al-

Received Dec. 11, 2000; revised May 25, 2001; accepted May 31, 2001.

This work was supported by National Institutes of Health Grant NS26150. We thank Drs. John Cooper and Elliot Elson for comments on this manuscript. Grady Phillips and Antoine Smith provided excellent technical assistance.

Correspondence should be addressed to Dr. Paul C. Bridgman, Department of Anatomy and Neurobiology, Washington University School of Medicine, Box 8108, 660 South Euclid Avenue, St. Louis, MO 63110. E-mail: bridgmap@pcg.wustl.edu. Copyright (C) 2001 Society for Neuroscience $0270-6474 / 01 / 216159-11 \$ 15.00 / 0$ though growth cones have more complex morphologies and behaviors, this model, in combination with the mechanism of substrate-cytoskeletal coupling (Suter et al., 1998), can be adapted to explain myosin II-driven growth cone advance (Heidemann and Buxbaum, 1998).

At least two isoforms of nonmuscle myosin II heavy chain (A and B) are present in neuronal tissue (Itoh and Adelstein, 1995). The B isoform is enriched in neurons and is concentrated as an ordered arrangement of mini-filaments in specific regions of the growth cone (Rochlin et al., 1995). Bipolar filaments can produce a contractile force when they interact with actin filaments of opposite polarities. This force could regulate the organization and tension on actin filaments and affect growth cone shape and traction. Previous studies that used drugs or antisense RNA to inhibit the function of myosins in neurons or neuroblastoma cells suggest a role for myosin II in outgrowth or growth cone motility (Lin et al., 1996; Ruchhoeft and Harris, 1997; Wylie et al., 1998). However, these studies also raise a number of questions regarding the specificity of the methodology that was used and the ability to identify and measure myosin II-dependent changes. To determine the exact role that myosin IIB plays in growth cone function, we used myosin IIB knock-out (KO) mice. Genetic ablation of myosin IIB has severe effects on development, including congenital hydrocephalus beginning at early embryonic stages (Tullio et al., 1997, 2001; Uren et al., 2000) and slowing of axonal outgrowth (Tullio et al., 2001). Slowed axonal outgrowth is consistent with effects on growth cone motility. Therefore, we tested the following hypotheses. (1) Myosin IIB regulates growth cone shape dynamics. (2) Myosin IIB regulates actin organization that is responsible for maintaining a spread morphology. (3) Myosin 
IIB-mediated contraction contributes to traction force generation.

\section{MATERIALS AND METHODS}

The targeted gene disruption of the nonmuscle myosin heavy chain B in mice has been described (Tullio et al., 1997). Superior cervical ganglion (SCG) neurons were selected for study because they grow rapidly and produce large, well spread growth cones when plated on laminin substrates. SCG explant cultures were grown from E17-E19 embryos or P0 mouse pups as described previously (Rochlin et al., 1995). Quantification of outgrowth rates from explants was as described previously (Tullio et al., 2001). Potential myosin IIB $\mathrm{KO}\left(\mathrm{B}^{-1-}\right)$ embryos and pups were selected on the basis of their abnormal morphology: an enlarged domeshaped cranium and short limbs. Their phenotype was confirmed by Western blotting. Southern blots then were used to distinguish between wild-type $\left(\mathrm{WT} ; \mathrm{B}^{+1+}\right)$ and heterozygous $\left(\mathrm{B}^{+/-}\right)$mice. Living cultures $12-18 \mathrm{hr}$ in vitro were visualized by phase contrast or differential interference contrast (DIC) microscopy. Images were taken by standard film photography or were recorded by video-enhanced methods at $5 \mathrm{sec}$ intervals and stored as digital image files. Quantitation of growth cone area and perimeter was done directly on digital images with IPLab (Scanalytics, Fairfax, VA). The growth cone images were traced by hand with a Wacom digital tablet. After some preliminary analysis we determined that comparisons done at 1 min intervals detected at least $90 \%$ of the changes. One minute intervals were used for subsequent analysis. In our analysis we identified every protrusion and retraction sequentially around the cone perimeter and calculated their areas in pixels. To convert this to real area, we used a calibration standard imaged with the same optics as for the recordings and calculated the image area in square micrometers. The leading edge was defined as the area of the cone perimeter from which expansion led to forward advance along a trajectory in line with the proximal neurite. Although this was sometimes difficult to discern in individual static images, replaying time-lapse sequences allowed us to define these areas at the measurement intervals by an interactive process.

Immunofluorescence and rhodamine phalloidin staining of growth cones were performed as described previously by using glutaraldehyde fixation (Rochlin et al., 1995). Images were collected by a cooled CCD camera (Photometrics, Tucson, AZ). To determine actin bundle density, we traced individual bundles as a series of lines on digital images. A bundle segment was defined as an individual line or the portion of a line originating from an intersection with a crossing or branching line. For quantitation of intensity, a flat field and background corrections were performed, and then intensity data were collected from the 16-bit images. The perimeter of individual growth cones was traced, and the average and peak intensities per pixel within the growth cone were calculated for each fluorochrome. Images were displayed as color overlays, using Adobe Photoshop (San Jose, CA).

Preparation and calibration of acrylamide gel substrates. To measure traction force, we grew neurons on thin, highly flexible laminin-coated acrylamide gels containing fluorescent beads (Pelham and Wang, 1999). The percentage of acrylamide $(3.75 \%)$ and $N, N$-methylene-bisacrylamide (BIS) cross-linker $(0.03 \%)$ selected for the experiments was determined by a set of preliminary tests. The percentages of acrylamide and BIS were varied until a combination was found that resulted in bead movement by growth cones. The preparation of gels was modified from published procedures (Pelham and Wang, 1999) to reduce the background fluorescence associated with out-of-focus beads. A thin 12-mmdiameter gel $(3.75 \%$ acrylamide and $0.03 \%$ BIS) without fluorescent beads was polymerized first on a $22 \times 40 \mathrm{~mm}$ coverslip. This was followed by overlaying another 22-mm-diameter gel of the same composition, but one containing fluorescent beads. Removal of the top round coverslips used for the formation of the two layers was done slowly, with the gels fully immersed in buffer. This prevented distortion of the soft gel surface and resulted in a very thin top layer of gel containing beads in the $12 \mathrm{~mm}$ center. All beads within the central ( $\sim 8 \mathrm{~mm}$ diameter) area were in a single focal plane, using a 0.75 numerical aperture $40 \times$ lens. Three methods of gel calibration were used. The first method was used to allow for direct comparison with published values (Pelham and Wang, 1997). Young's modulus of the 10 and $3.75 \%$ acrylamide gels was calculated from large gel loops that were stretched by weights while in a solution bath. In the second method, $3.75 \%$ acrylamide gel loops were stretched and then relaxed on a device containing a force transducer (Wakatsuki et al., 2000). The device can stretch the gels for a known amount and detect the force on the gels as a function of the distance stretched. Initially, the gels were stretched $7 \mathrm{~mm}$ (inner diameter). From this initial condition the gels were stretched to $10.5 \mathrm{~mm}$ and then gradually released back to $7 \mathrm{~mm}$. A continuous readout of force versus the distance stretched was obtained for each gel. During the stretching the force increased linearly with distance. Relaxation showed the same relationship. Thus the gels are elastic. Then the gels were removed from the force transducer device and were stained with an aqueous solution of Coomassie blue so that they were clearly visible. The gels were restretched to $7 \mathrm{~mm}$, and the crosssectional area was measured. The gels were submerged in PBS at all times. Young's modulus was calculated by using the equation: $Y=$ $(F / A) /(\Delta L / L)=$ slope of the force versus the distance stretched in the plot $\times$ original length/cross-sectional area. Young's modulus from the first method for the $10 \%$ gels was $4000 \mathrm{~N} / \mathrm{m}^{2}$. For the $3.75 \%$ acrylamide gels the second method of calibration gave a Young's modulus lower than that of the first $\left(150 \mathrm{vs} 8 \mathrm{~N} / \mathrm{m}^{2}\right)$. The second method was more reproducible.

In the third method of calibration the shear compliance was measured with a calibrated glass needle to deform the same thin double-layer acrylamide sheets on which the cells were grown. The needle was calibrated by the method of Lee et al. (1994) and was calculated to exert a force of $0.216 \mathrm{dyn} / \mathrm{cm}$. The distance the beads were displaced was measured as a function of the distance from the point of application of the force, which was taken as the midpoint between the initial and final positions of the needle tip. The distances of bead displacement were measured as a component of distance along the direction of force application. This was required because the tip of the needle indented the gel, displacing the beads slightly toward the needle tip. These data were extrapolated linearly to find the displacement at zero distance (from the midpoint position of the needle tip). The displacement was found to be $5.25 \mu \mathrm{m}$. Thus our standard gel required $40.4 \mu$ dyn to displace a bead 1 $\mu \mathrm{m}$. This value would depend on the thickness of the gel, so we maintained a constant gel thickness by using the same volume of gel. Regional variations in shear compliance were insignificant within the central region $(\sim 6-8 \mathrm{~mm}$ diameter) of the gel. Therefore, we recorded only from growth cones in this area.

To insure that the gels were the same thickness and that other factors were not affecting the measurements, we tested each in the region of recordings with the calibrated needle. By overlaying images of fluorescent beads before and after deformation by the calibrated needle, we produced a bead displacement map (Oliver et al., 1995; Dembo et al., 1996). By superimposing displacement maps from different experiments, we could compare the shear compliance of different gels and, if required, normalize the data for any differences. We also tested whether the two gel layers acted as a bonded unit or slid past one another. We formed the bottom layer of gel with fluorescent beads and then formed the top layer without beads. Using the calibrated needle to deform the top bead-free gel layer, we observed a displacement of beads in the upper focal plane (adjacent to the bead-free layer) along the direction of force application. This suggests that the two layers acted as a single bonded unit.

Time-lapse images were taken at 10 or $20 \mathrm{sec}$ intervals with a cooled CCD camera. The growth cones and beads were imaged simultaneously with phase contrast and fluorescence by using a Zeiss $40 \times 0.75$ numerical aperture lens. For quantitation of bead movement induced by filopodia, all interactions that caused bead movements for $>3$ frames $(60 \mathrm{sec})$ of a time-lapse series were analyzed. Because some time-lapse series ended before the full release of beads, it was not always possible to determine the full length of filopodia-bead interaction.

\section{RESULTS}

SCG neurons from myosin IIB KO animals and normal littermates were grown in cell culture. As reported previously, outgrowth rates of axons from explants taken from the $\mathrm{KO}$ animals were decreased compared with outgrowth from WT explants (Tullio et al., 2001). When they were grown on laminin substrates, the average decrease in outgrowth rate was $\sim 32 \%$. In general, $\mathrm{KO}$ growth cones in fixed cultures were also smaller and more irregularly shaped than those of the WT.

\section{KO growth cones have altered shape-changing dynamics}

First, we asked whether KO growth cones showed alterations in shape dynamics. If cortical tension is decreased, then it is possible that both forms of actin-based protrusive structures, filopodia and 

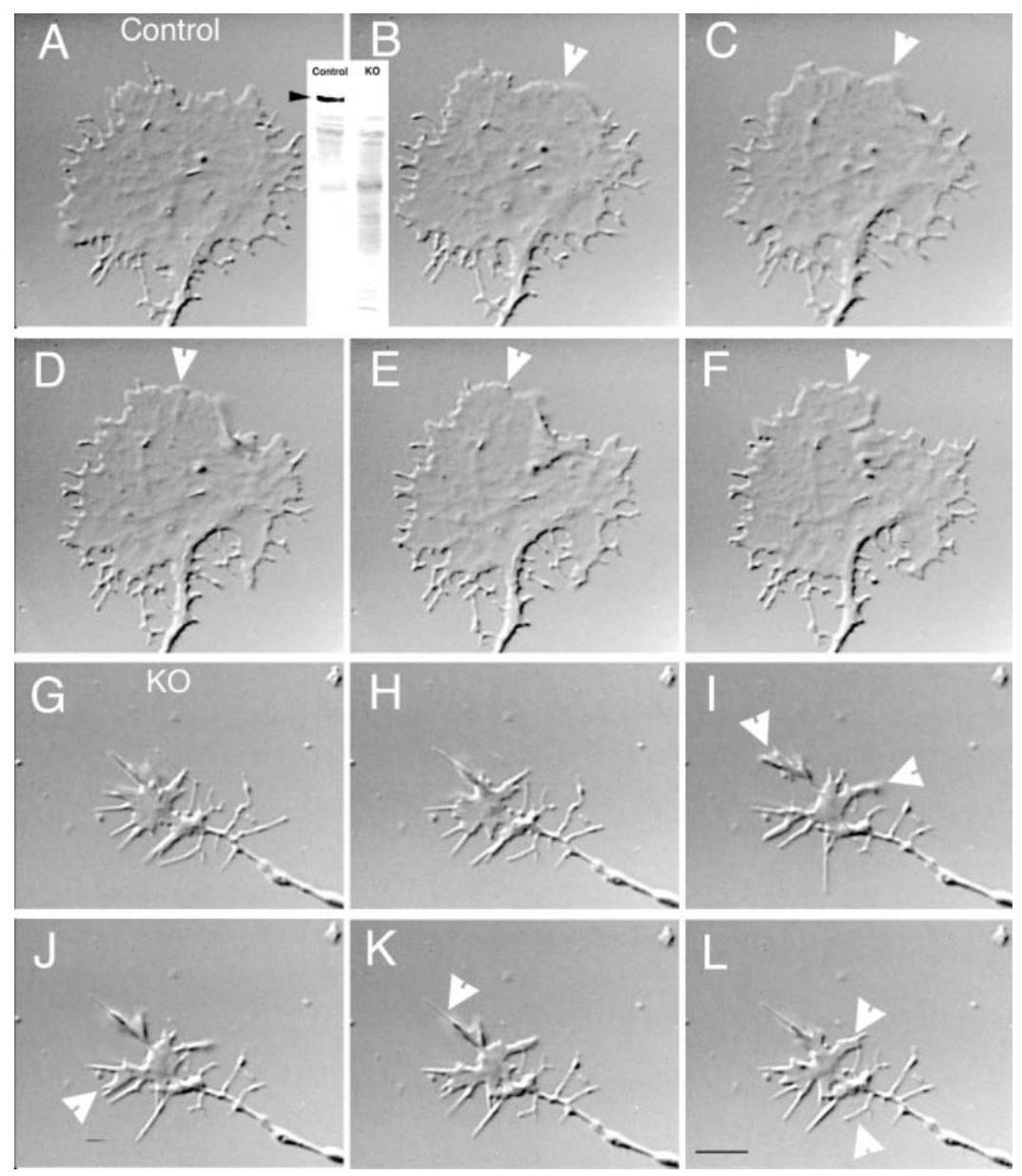

Figure 1. Examples of video-enhanced DIC time-lapse sequences taken from WT (Control; $A-F$ ) and knock-out SCG growth cones $(K O ; G-L)$. Protrusions (arrowheads) in the WT occur mainly at the leading edge. In contrast, protrusions (arrowheads) in the KO occur both at the leading edge and at other areas around the perimeter of the cone. Frames are at $15 \mathrm{sec}$ intervals. $A, B$, Inset, Immunoblot for myosin IIB in brains from the mice that were used for these cultures. A $200 \mathrm{kDa}$ band specific for myosin IIB is seen only in the control. Scale bar, $6 \mu \mathrm{m}$. lamellipodia, may form at different rates and have different sizes. So that growth cone filopodia and lamellipodia dynamics can be analyzed accurately, high-resolution movies of growth cone are required. Therefore, video-enhanced DIC microscopy was used to make time-lapse observations on growth cones from $\mathrm{KO}$ and WT animals (Fig. 1). When we viewed the time-lapse movies at different speeds, it was apparent that the growth cones from $\mathrm{KO}$ animals made more frequent changes in shape. To quantify the dynamics, we digitized individual images. Growth cone outlines were compared between time points to determine regions of new protrusion (extension) and retraction of the growth cone perimeter. Because it was often difficult to classify these areas as either filopodia or lamellipodia, especially early in their formation, we define these only in terms of protrusion or retraction area. Protrusion and retractions were identified, measured for size, and numbered by starting at the base of the cone (immediately adjacent to the right side of the neurite) and going sequentially around the perimeter until reaching the left side of the neurite (Fig. $2 A, B$ ). Typically, during a single 1 min interval (Fig. $2 C, D$ ) a $\mathrm{KO}$ cone showed smaller areas but more frequent protrusions and retractions per length of perimeter than a WT cone. When we view the data as a time series (Fig. $2 E, F$ ), it is apparent that the decreased size and greater frequency of protrusions and retractions observed at a single time point are consistent properties of the $\mathrm{KO}$ growth cone.

These data suggest that the greater frequency and smaller size of protrusions and retractions contribute to the more complex shapes and different dynamics of KO cones. However, growth cones are highly variable in their behavior. Normally, cones will undergo periods of rapid advance, followed by periods of less rapid advance or stalling. These changes in advance are associated with changes in shape. To test whether our prediction about $\mathrm{KO}$ growth cone behavior is correct in a more rigorous way, we first calculated the average size of individual protrusions and retractions at $1 \mathrm{~min}$ intervals from a total of $80 \mathrm{~min}$ of recording from each group, WT and KO (eight different cones for each). The mean size of protrusions that formed each minute for WT cones was $2.4 \pm 0.15 \mu \mathrm{m}^{2}(\mathrm{SEM} ; n=612)$, whereas that of $\mathrm{KO}$ cones was $1.4 \pm 0.08 \mu \mathrm{m}^{2}(\mathrm{SEM} ; n=708)$. The difference was highly significant $(t$ test; $p<0.001)$. The mean size of retractions that formed each minute for WT cones was $1.3 \pm 0.05 \mu \mathrm{m}^{2}$ $(\mathrm{SEM} ; n=875)$, and that of $\mathrm{KO}$ cones was $0.9 \pm 0.03 \mu \mathrm{m}^{2}(\mathrm{SEM}$; $n=778)$. The difference in mean retraction area was also highly significant ( $t$ test; $p<0.001$ ). Thus both protrusions and retractions are larger in WT cones.

Note that for both cases the average size of protrusions is greater than the average size of retractions. For a cone to maintain its size and shape, the protrusion and retraction area must be approximately equivalent over a given time period. However, for growth cones the area of retraction will always be less than that of protrusions because the neurite forms as a trailing process. The total area of protrusion and retraction in a given time period will depend on both the size and number of protrusions and retractions. Therefore, we also analyzed eight wild-type and eight $\mathrm{KO}$ cones for net area of protrusion and retraction formed at $1 \mathrm{~min}$ intervals. Wild-type cones had a significantly larger area of pro- 

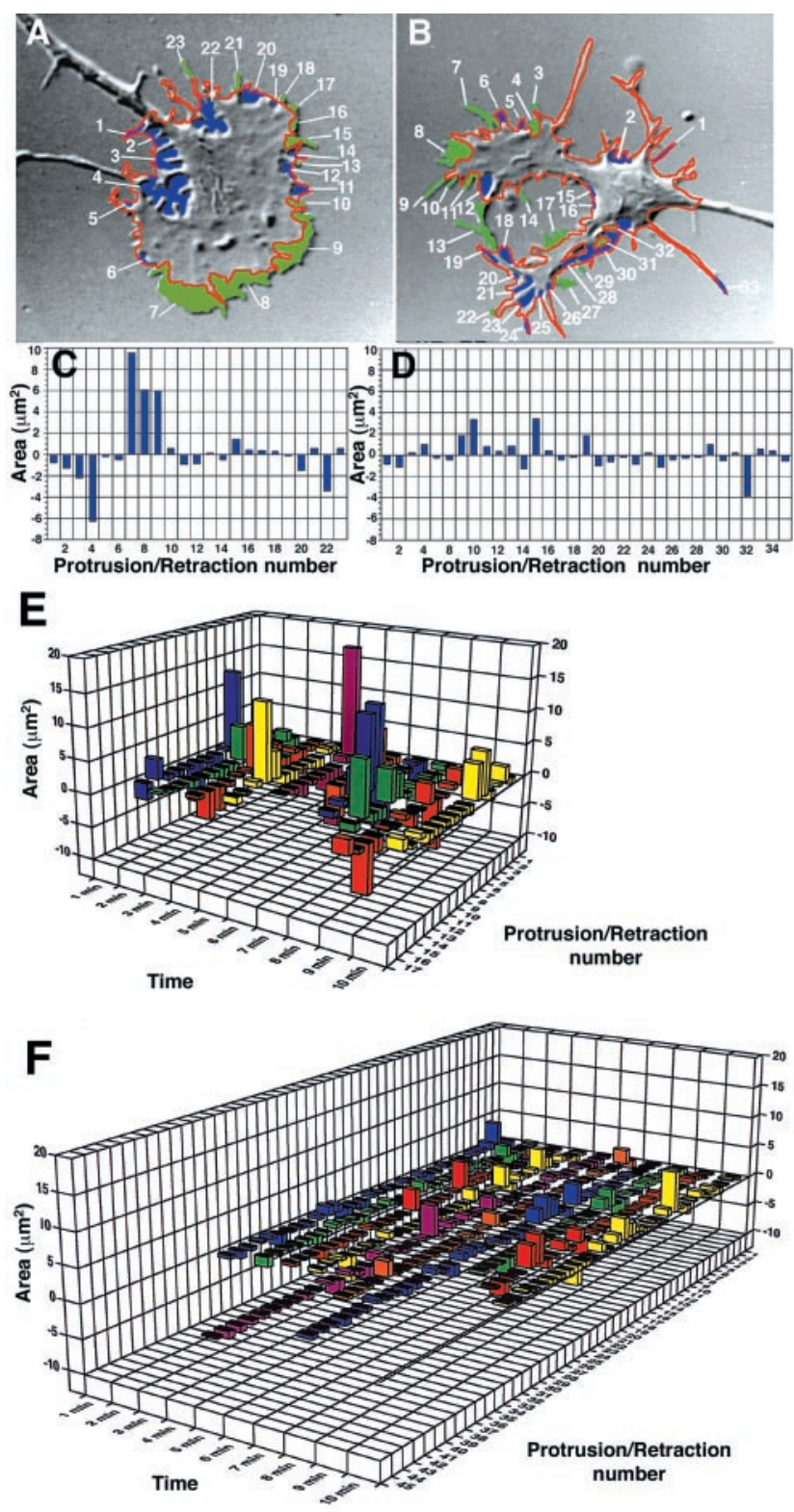

Figure 2. An example of the analysis of protrusion and retraction in growth cones from WT $(A, C, E)$ and myosin IIB KO $(B, D, F)$ animals. $A, B$, The perimeter of the growth cone traced from the previous time point is indicated by the red outline. Areas of protrusions are indicated in green overlays, whereas areas of retractions are indicated in blue. The sequential numbering around the perimeter of the cones indicates each area of either protrusion or retraction that had been identified in a $1 \mathrm{~min}$ time interval and then used as a single time point for plots of protrusion/ retraction number versus the area shown in $C$ and $D$ and for the multiple time points shown in $E$ and $F$. $C$ represents the WT cone shown in $A ; D$ represents the KO cone shown in $B$. In $C$ and $D$ the positive values indicate protrusion areas, and the negative values indicate retraction areas. $E, F$, Examples of the time series analysis of growth cone protrusion and retraction taken from time-lapse records of WT (as in $A$ ) and myosin IIB KO (as in $B$ ) cones of approximately equal area. Note that, as in $C$ and $D$, protrusion areas are indicated as positive values (on the $z$-axis), whereas retraction areas are indicated as negative values. The sequential numbering around the cone perimeter for each time point is indicated on the $y$-axis. A single 1 min time point (identified with different colors; $x$-axis) in $E$ or $F$ represents an individual set of area measurements as shown in $C$ or $D$. The sizes of protrusions and retractions tend to be larger in the WT cone but occur less frequently over the $10 \mathrm{~min}$ period. Thus the differences in area and frequency of protrusion and retraction that are observed in $C$ and $D$ persist over time. trusions per minute than $\mathrm{KO}$ cones $(\mathrm{WT}=20 \pm 1.3$ vs $\mathrm{KO}=$ $13 \pm 0.8 \mu \mathrm{m}^{2} ; t$ test; $\left.p<0.002\right)$. There was no significant difference in area of retractions. This may contribute to the smaller size of the KO cones (Table 1). The average frequency of protrusions and retractions was not significantly different from that observed in $\mathrm{KO}$ cones. However, because the $\mathrm{KO}$ cones were, on average, less than one-half the area of the wild-type cones and because the ability to protrude and retract the peripheral margin partially depends on the size of the cone because of more available perimeter, we also compared cones that were normalized for differences in size (Table 1). If one takes into account the difference in size, then KO cones show significantly greater rates of both protrusion and retraction. From the average areas of protrusion and retraction per minute, one can calculate the net area of expansion for both types of cones (area of protrusion - area of retraction $=$ net area of expansion). Because the advance of the growth cone is coupled to neurite formation, this number is proportional to the increase in neurite length. For WT cones the net area of expansion was $7.2 \mu \mathrm{m}^{2} / \mathrm{min}$, and for $\mathrm{KO}$ cones the area was $3.3 \mu \mathrm{m}^{2} / \mathrm{min}$. The ratio of WT-to-KO expansion rate (2.2) was very close to the ratio of cone size (2.1), suggesting that the expansion rate differences also give rise to the difference in cone size. The slightly more than twofold greater rate of expansion in WT cones is larger than the difference that was observed in outgrowth rate between wild-type and KO SCG neurons (Tullio et al., 2001), consistent with the idea that a greater rate of expansion is needed to maintain cone size.

Next we wanted to know whether or not the KO growth cones were as efficient in their ability to spread in the direction of advancement. We reasoned that alterations in the ability to form protrusions in the direction of growth also might affect outgrowth rates. To address this question, we analyzed time-lapse images of growth cones for location of protrusion and retraction. Inspection of the shape, area, and location of the protrusions around the perimeter of the cones that were analyzed from time series revealed that large protrusions occurred more frequently at the leading edge in cones from both WT and KOs. However, protrusions in the KO cones seemed to be less "focused" at the leading edge and were more spike-like compared with WT. To test whether this was accurate, we summed the areas of protrusions at the leading edge (see Materials and Methods for how the leading edge was defined) at 1 min intervals and compared them with the areas of protrusion in nonleading edge regions (Table 1). The area of protrusion at the leading edge in WT growth cones was significantly greater than that observed in $\mathrm{KO}$ cones. In addition, the area of leading edge protrusion in WT cones was slightly greater than twice the area of protrusion on the sides/rear of WT cones. In contrast, the area of protrusion at the leading edge of $\mathrm{KO}$ cones was slightly less than the sides/rear of KO cones. The area of protrusion on the sides/rear of WT cones was not significantly different from that in $\mathrm{KO}$ cones. A similar location-specific difference in the area of retractions was not observed between WT and KO cones. Net expansion occurred at the leading edge of both WT and KO cones, but the area of leading edge expansion was much larger for the WT cones. Similarly, net retraction occurred on the sides/rear of both WT and KO cones, but again the area of retraction was greater for WT cones. Thus WT cones maintained a more polarized structure via the formation of larger areas of expansion at the leading edge and larger areas of retraction on the sides/rear of the cone. Although the KO cones managed to remain somewhat polarized, misoriented protrusive areas (not in the direction of advancement) formed at approxi- 


\begin{tabular}{|c|c|c|c|c|c|}
\hline$\#$ & $\begin{array}{l}\text { Average area of } \\
\text { protrusion/min } \\
\text { (retractions/min) } \\
\text { at leading edge } \\
\left(\mu \mathrm{m}^{2}\right)\end{array}$ & $\begin{array}{l}\text { Average area of } \\
\text { protrusion } / \mathrm{min} \\
(\text { retractions } / \mathrm{min}) \\
\text { at sides/rear }\left(\mu \mathrm{m}^{2}\right)\end{array}$ & $\begin{array}{l}\text { Protrusion (retraction) } \\
\text { rate (number per min) }\end{array}$ & $\begin{array}{l}\text { Total cone area } \\
\left(\mu \mathrm{m}^{2}\right)^{*}\end{array}$ & $\begin{array}{l}\text { Normalized protrusion } \\
\text { (retraction) rate } \\
\text { (number per min) }\end{array}$ \\
\hline \multicolumn{6}{|c|}{ Wild type } \\
\hline 1 & $\begin{array}{l}14.1 \pm 0.7 \\
(3.9 \pm 1.7)\end{array}$ & $\begin{array}{c}18.4 \pm 0.6 \\
(16.8 \pm 1.2)\end{array}$ & $20.9(23.6)$ & 290 & $7.2(8.1)$ \\
\hline 2 & $\begin{array}{c}9.3 \pm 0.8 \\
(1.5 \pm 0.5)\end{array}$ & $\begin{array}{c}3.8 \pm 0.5 \\
(10.5 \pm 1.2)\end{array}$ & $6.6(8.0)$ & 98 & $6.7(8.2)$ \\
\hline 3 & $\begin{array}{c}6.0 \pm 0.2 \\
(1.9 \pm 0.7)\end{array}$ & $\begin{array}{l}7.7 \pm 0.6 \\
(11 \pm 1.6)\end{array}$ & $10.7(11.7)$ & 196 & $5.5(6)$ \\
\hline 4 & $\begin{array}{l}11.9 \pm 0.2 \\
(3.5 \pm 0.7)\end{array}$ & $\begin{array}{c}4.5 \pm 0.3 \\
(6.6 \pm 0.9)\end{array}$ & $8.8(8.9)$ & 145 & $6.1(6.1)$ \\
\hline 5 & $\begin{array}{l}23.5 \pm 1.3 \\
(2.4 \pm 0.8)\end{array}$ & $\begin{array}{l}3.9 \pm 1.1 \\
(6.5 \pm 1)\end{array}$ & $4.8(8.1)$ & 181 & $2.7(4.5)$ \\
\hline 6 & $\begin{array}{l}12.9 \pm 1.7 \\
(7.9 \pm 2)\end{array}$ & $\begin{array}{c}2.6 \pm 0.5 \\
(11.6 \pm 1.7)\end{array}$ & $4.5(7.5)$ & 131 & $3.4(5.7)$ \\
\hline 7 & $\begin{array}{l}16.7 \pm 1.5 \\
(4.9 \pm 1.7)\end{array}$ & $\begin{array}{c}12.4 \pm 1 \\
(12.9 \pm 1.7)\end{array}$ & $8.2(10.1)$ & 227 & $3.6(4.5)$ \\
\hline 8 & $\begin{array}{l}12.5 \pm 1.2 \\
(1.2 \pm 0.7)\end{array}$ & $\begin{array}{c}3.2 \pm 0.5 \\
(3.9 \pm 2.2)\end{array}$ & $4.3(3.8)$ & 108 & $4.0(3.5)$ \\
\hline Mean & $\begin{array}{l}13.5 \pm 1.9^{* *} \\
(3.4 \pm 0.8)\end{array}$ & $\begin{array}{l}7.1 \pm 2 \\
(10 \pm 1.5)\end{array}$ & $\begin{array}{c}8.6 \pm 1.7 \\
(10.2 \pm 2.1)\end{array}$ & $172 \pm 23^{* * *}$ & $\begin{array}{c}4.9 \pm 0.5 * * * * \\
(5.8 \pm 0.6)^{* * * * *}\end{array}$ \\
\hline \multicolumn{6}{|c|}{ Myosin IIB KO } \\
\hline 1 & $\begin{array}{c}5.8 \pm 0.5 \\
(1.5 \pm 0.3)\end{array}$ & $\begin{array}{c}8.6 \pm 0.3 \\
(11.5 \pm 0.8)\end{array}$ & $14.5(19)$ & 122 & $11.9(15.6)$ \\
\hline 2 & $\begin{array}{c}3.8 \pm 0.2 \\
(0.9 \pm 0.3)\end{array}$ & $\begin{array}{c}3.7 \pm 0.2 \\
(3.4 \pm 0.4)\end{array}$ & $9.6(7.2)$ & 68 & $14.1(10.6)$ \\
\hline 3 & $\begin{array}{r}5.6 \pm 0.41 \\
(2.2 \pm 0.3)\end{array}$ & $\begin{array}{c}3.2 \pm 0.2 \\
(3.8 \pm 0.2)\end{array}$ & $7.8(9.4)$ & 49 & $15.9(19.2)$ \\
\hline 4 & $\begin{array}{c}4.6 \pm 0.4 \\
(0.6 \pm 0.2)\end{array}$ & $\begin{array}{c}2.3 \pm 0.3 \\
(4.4 \pm 0.9)\end{array}$ & $7.6(5.6)$ & 56 & $13.6(10)$ \\
\hline 5 & $\begin{array}{c}6.5 \pm 0.6 \\
(4.6 \pm 1.1)\end{array}$ & $\begin{array}{c}11.3 \pm 1 \\
(12.3 \pm 0.9)\end{array}$ & $10.2(8.4)$ & 71 & $14.4(11.8)$ \\
\hline 6 & $\begin{array}{c}3.1 \pm 0.5 \\
(2.3 \pm 0.6)\end{array}$ & $\begin{array}{l}7.3 \pm 0.5 \\
(9.1 \pm 1.3)\end{array}$ & $10.5(13.7)$ & 97 & $10.8(14.1)$ \\
\hline 7 & $\begin{array}{c}8.8 \pm 1.9 \\
(1.4 \pm 0.9)\end{array}$ & $\begin{array}{c}8.2 \pm 0.5 \\
(6.2 \pm 1.1)\end{array}$ & $7.6(8.6)$ & 49 & $15.5(17.6)$ \\
\hline 8 & $\begin{array}{l}11.7 \pm 1.2 \\
(3.9 \pm 1.0)\end{array}$ & $\begin{array}{c}12.5 \pm 1 \\
(11.3 \pm 1.5)\end{array}$ & $8.4(14.1)$ & 147 & $5.7(9.6)$ \\
\hline Mean & $\begin{array}{l}6.2 \pm 1^{* *} \\
(2.2 \pm 0.5)\end{array}$ & $\begin{array}{c}7.1 \pm 1.4 \\
(7.8 \pm 1.3)\end{array}$ & $\begin{array}{c}9.5 \pm 0.7 \\
(10.8 \pm 1.6)\end{array}$ & $82 \pm 13^{* * *}$ & $\begin{array}{c}12.7 \pm 11^{* * * *} \\
(13.6 \pm 1.3)^{* * * * *}\end{array}$ \\
\hline
\end{tabular}

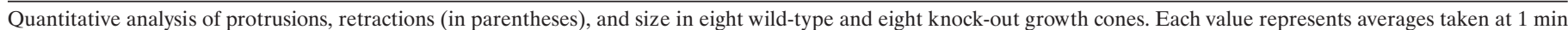

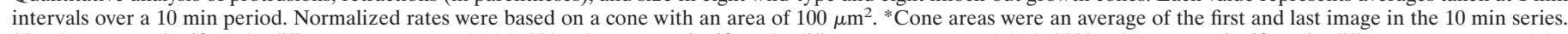

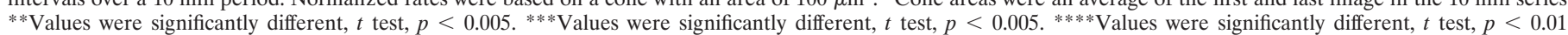
$* * * * *$ Values were significantly different, $t$ test, $p<0.001$.

mately the same rate as at the leading edge and constantly had to be retracted to maintain a somewhat polarized structure. The inability to maintain a highly polarized structure efficiently was associated with a more irregular cone shape and variability over time. It also may contribute to the slower outgrowth rates.

\section{Actin organization is abnormal in $\mathrm{KO}$ growth cones}

Because of the alterations in the dynamics of actin-containing structures and the smaller size of the KO cones, we wanted to know whether the actin filament organization in $\mathrm{KO}$ growth cones was altered. We reasoned that myosin IIB might be mainly responsible for cortical tension and maintaining a spread mor- phology in the cone. It may do this by its ability to form or stabilize actin bundles. Rhodamine phalloidin staining was used to compare the growth cone actin organization. In normal cones the actin bundles extend through the "palm" or marginal zone of the cone in various orientations (Fig. 3). These are the bundles that normally are associated with the most concentrated foci of myosin IIB staining (Rochlin et al., 1995), suggesting that they may be under tension. Actin was organized abnormally in the $\mathrm{KO}$ cones; i.e., distinct actin bundles were reduced significantly in density from the growth cone palm $(\mathrm{WT}=9.5 \pm 0.63$ bundle segments $/ 10 \mu \mathrm{m}^{2}$ vs KO $=6.1 \pm 0.99$ bundle segments $/ 10 \mu \mathrm{m}^{2}$; 

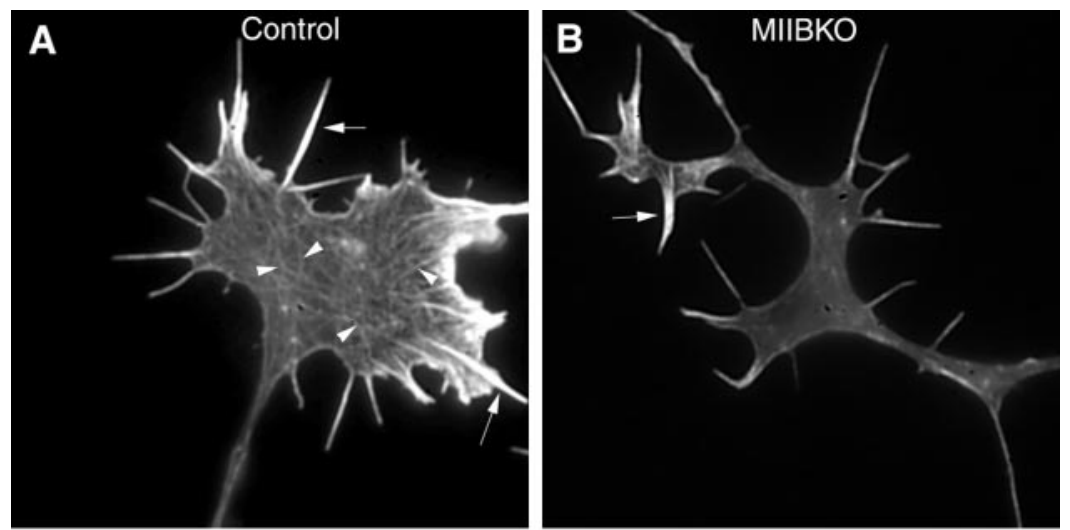

Figure 3. F-actin organization is abnormal in growth cones from myosin IIB KO animals. $A$, The distribution of F-actin that is stained with rhodamine phalloidin in a control (WT) growth cone. Note that the palm of the cone contains numerous actin bundles (arrowheads). $B-D$, The distribution of F-actin in three $\mathrm{KO}$ cones is shown. Although actin bundles are still present in filopodia (arrows), they are absent or decreased in the narrowed palm of the cone. Scale bar, $4 \mu \mathrm{m}$.
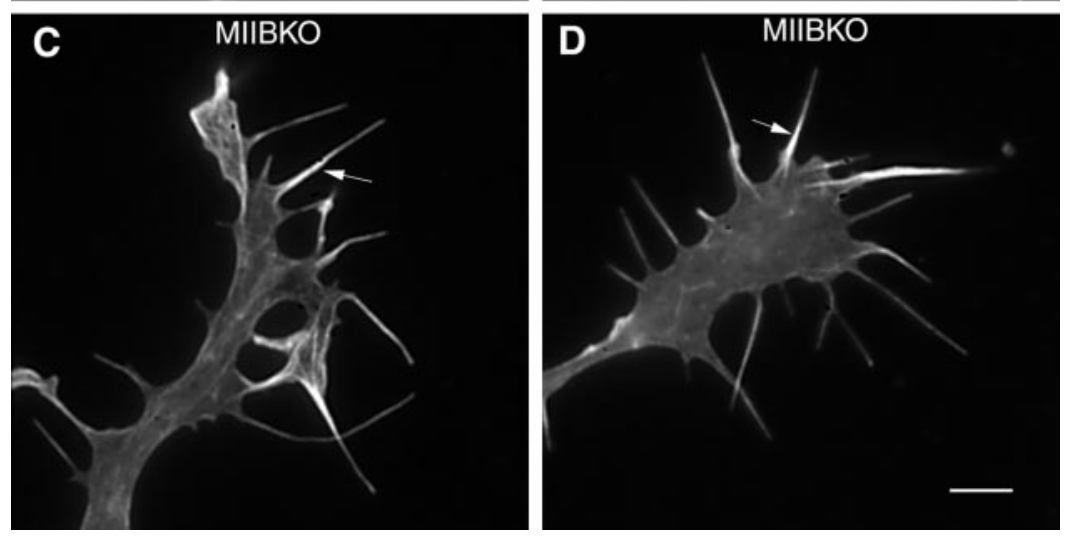

$t$ test; $p<0.01$ ). Bundles were still present in most filopodia (Fig. $3)$. This suggests that myosin IIB contributes either to the formation of the actin bundles in the palm or to their stabilization. The decreased density of these bundles may be partially a result of the absence of broad areas of lamellipodia in the KO cones, because actin bundles in lamellipodia move by retrograde flow into and then consolidate in the growth cone palm. Myosin IIB is not necessary for the formation of actin bundles that form the core of filopodia, because they appear normal.

\section{KO filopodia produce less traction force}

Although the alterations in the ability to organize or maintain a polarized shape may be responsible for the decreased rate of advancement in the $\mathrm{KO}$ cones, it is possible that other factors contribute. Myosin IIB could contribute to the generation of traction force via a contractile mechanism. Thus we wanted to know whether traction force was altered in KO growth cones. This is a difficult question to address. Although the total amount of traction force generated by individual growth cones has been measured (Lamoureux et al., 1989), direct comparisons using this method would be difficult in this case. This is because the differences in growth cone sizes between WT and $\mathrm{KO}$ will contribute to the total traction force, and it is not clear whether there are differences in the relative contributions of filopodia and lamellipodia to the total force. For this reason we needed to find a method that could be used to measure the force developed by an individual filopodium or discrete areas of lamellipodia. This has never been done in growth cones. We adapted a method that has been used by Wang and colleagues to study forces exerted by other cell types (Pelham and Wang, 1999). This method uses compliant acrylamide gels containing fluorescent beads. The movement of the beads is used to monitor local forces exerted on the substrate.
To adapt this method, we first had to determine whether conditions could be found that would allow for measurements of growth cone traction forces. Growth cones produce much less force than other cell types (Lamoureux et al., 1989), so this is not a trivial task. If WT neurons are grown on laminin-coated acrylamide sheets $(10 \%)$ that are deformed by other cell types, they grow normally but do not deform the sheets, as indicated by the lack of any detectable effects on outgrowth, growth cone morphology, or bead movements (data not shown). If the acrylamide sheets $(3.75 \%)$ are made much more compliant ( $>26$ times), the neurons still grow but at reduced rates. In addition, growth cones tended to spread less on these more highly compliant sheets. Both changes are consistent with the force-producing capabilities of the growth cone interacting with a substrate of sufficient compliance to be altered during growth. In time-lapse sequences taken of WT growth cones growing on the more compliant gels, fluorescent beads were observed to move when the cones were directly over the beads, indicating that they were exerting sufficient force on the substrate to deform it (Fig. $4 A-H$ ). Although most growth cones produced bead displacement, the degree of displacement was variable, and many of the filopodial and lamellipodial extensions did not cause bead movement. Possibly, this was because they were not adherent. The bead density was not high and the forces were weak, so filopodia or lamellipodia caused movements only when directly over a bead. These interactions were relatively rare. However, these conditions allowed for measurements of force exerted by an individual filopodium or discrete areas of lamellipodia.

The character and degree of bead movement differed between lamellipodia and filopodia in WT cones. Lamellipodia caused more frequently detected movements of beads generally toward the growth center or palm because of their larger area. However, 

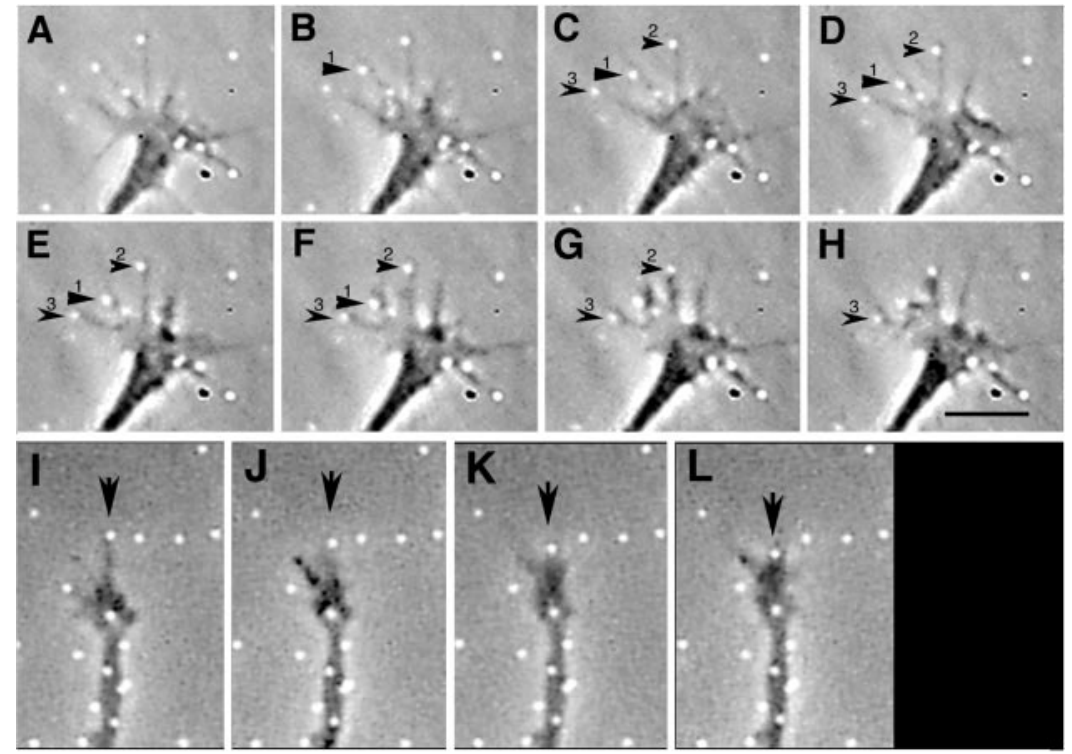

M

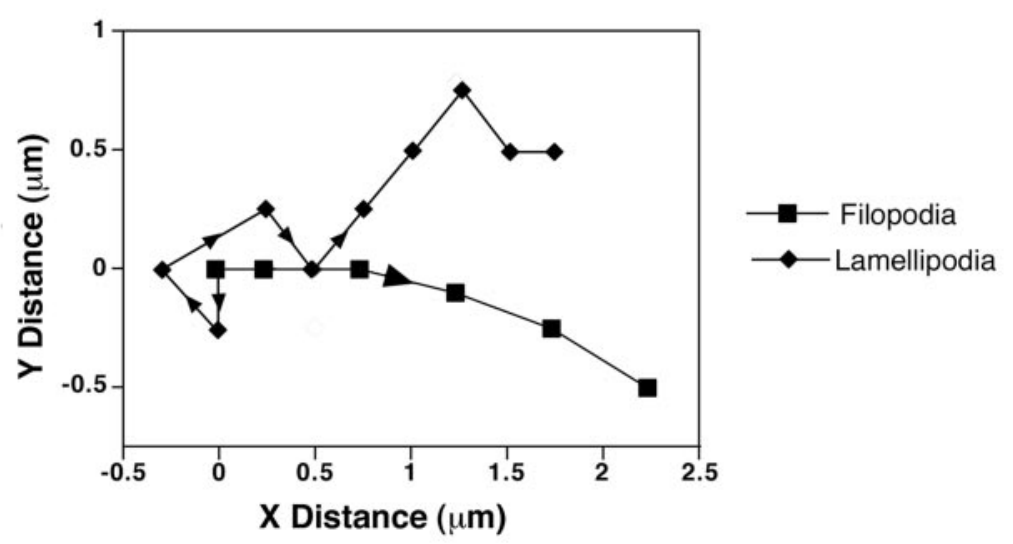

Figure 4. Interaction of growth cone filopodia and lamellipodia with the $3.75 \%$ acrylamide gel surface causes displacement of fluorescent beads (white dots) embedded in the gel. $A-H$, Filopodia in a control (WT) growth cone cause displacement of three (1-3) adjacent beads at different time intervals. The beads move (arrowheads) toward the growth cone palm. Bead 1 appears to move initially $(B-D)$ without filopodia shortening. Later, the filopodia appear to move laterally $(E)$ and then shorten (note increased phase density in $F-H$ ). Both beads 2 and 3 appear to move as the filopodia shorten. Time between frames is $20 \mathrm{sec} . I-L$, Bead movement in response to filopodial shortening in a $\mathrm{KO}$ growth cone. A single bead (arrowhead) moves toward the growth cone palm as the filopodia appear to shorten. Time between frames was $20 \mathrm{sec}$, except between $I$ and $J$ when the time was $40 \mathrm{sec} . M$, Comparison of bead movement induced by WT filopodial or lamellipodial interaction. Both examples start as 0,0 coordinates. Points indicate the bead centroid. A straight line along the $x$-axis would be parallel with the neurite. Positive values along the $x$-axis indicate movement toward the growth cone palm. In response to the lamellipodium a bead first moves to the side and then is pushed out (negative value along $x$-axis). Then the bead is pulled inward toward the growth cone palm but also moves laterally. In response to a filopodium a bead moves inward toward the growth cone palm with less lateral movement. Scale bar (in $H$ ): $9.5 \mu \mathrm{m}$. the movements were less in peak magnitude and more complicated in direction than those induced by filopodia (Fig. 4M). Filopodia at the front of a cone induced bead movements essentially along a single axis directed toward the growth cone palm. This made the comparison between different cells less complicated. Filopodia toward the rear of a cone or extending from the sides of a newly formed neurite usually showed a different behavior. The bead movement was often oscillatory and associated with small changes in filopodial length. Movement of the bead toward the cone center or neurite was associated with shortening of the filopodia, whereas movement away was associated with an increase in filopodial length. In contrast, bead movements induced by WT filopodial interactions at the front of a cone were rarely oscillatory. They involved persistent movements in a single direction (toward the cone center) that lasted tens of seconds to minutes (Fig. 4A-H). They sometimes occurred without detectable change in filopodial length or in rare cases (two) an increase in length. However, most often the bead movement was associated with a decrease in filopodial length and an increase in phase density proximal to the bead. This suggests that a filopodial contraction was responsible for the bead movement.

Because growth cones from the $\mathrm{KO}$ animals were smaller and rarely showed lamellipodia, the comparison of bead movement between $\mathrm{KO}$ and WT was done only between filopodial and bead interactions toward the front of cones. KO filopodia also caused bead movement concurrent with filopodia shortening (Fig. 4I-L). Plots of force versus time for an individual filopodium (Fig. 5) could be made from the calibrated gels. Filopodia of both WT and $\mathrm{KO}$ growth cones showed variable degrees of peak traction force. Surprisingly, the filopodia of $\mathrm{KO}$ cones could produce peak forces equivalent to those of WT cones, but they did so infrequently. The average amount of peak force per filopodium was reduced significantly in the KO cones, WT $=97 \pm 8.5 \mu$ dyn, SEM, $n=13$ from 8 cones; $\mathrm{KO}=66 \pm 11.1 \mu \mathrm{dyn}, \mathrm{SEM}, n=11$ from 9 cones; $t$ test; $p<0.05)$.

Although we measured the shear compliance of each gel in the region from which the recordings were taken, we wanted to address further the possibility that regional variation in compliance was influencing the results. Therefore, in one experiment we labeled WT neurons with an orange-red fluorescent dye (Cell Tracker orange) and KO cells with a green fluorescent dye (Cell Tracker green) and then plated them on the same acrylamide gel. We observed one WT filopodia interaction with a bead. The peak force was $160 \mu$ dyn. Immediately adjacent, we observed two KO filopodial interactions with beads in separate cones. The peak forces were 74 and $61 \mu \mathrm{dyn}$, respectively. Thus, the results are 


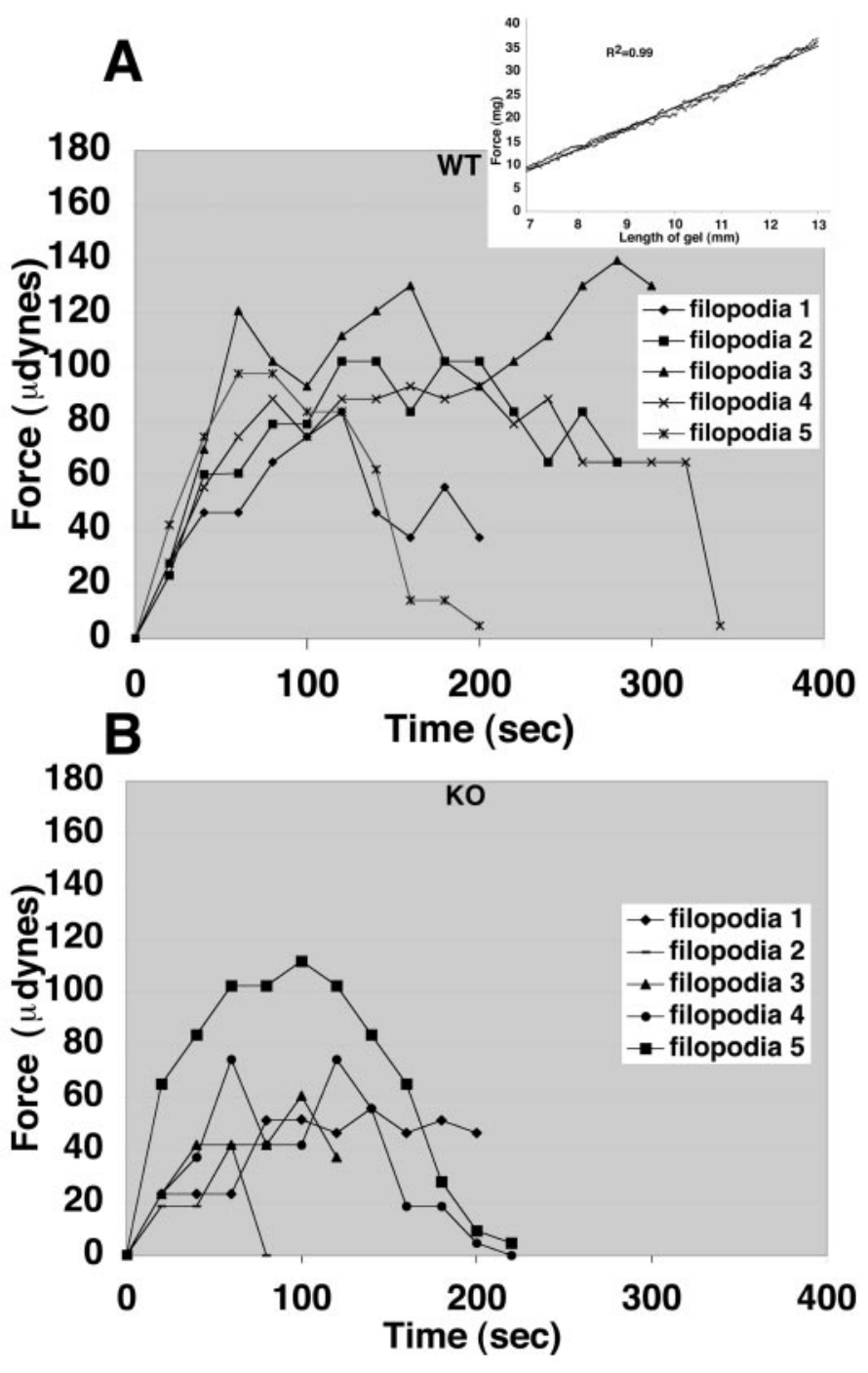

Figure 5. Examples of the change in force over time because of individual filopodial interactions with the gel substrate. Inset, The macroscopic elastic property of a $3.75 \%$ acrylamide gel is shown. The two traces indicate the force-versus-length relationship during gel stretching and relaxation. The curves are essentially the same. The least-squares best fit line through the data is indicated. $A$, The force exerted by filopodia from WT growth cones is depicted. The force tends to increase quickly (approximately half-maximal by $50 \mathrm{sec}$ ) and then stabilizes or decreases. $B$, The force exerted by filopodia from KO growth cones is shown. The force tends to develop slower and usually reaches a lower maximum value before decreasing. However, in filopodia number 5 the force-versus-time relationship is very similar to that seen in WT cones.

consistent with the differences in the peak forces exerted by WT and KO filopodia when cells are grown on separate gels.

Because we had observed an increase in the rates of both protrusion and retraction in $\mathrm{KO}$ cones, it is possible that filopodial lifetimes were reduced. Therefore, we also wanted to know whether the time length of force production by filopodia might be affected. In addition to the differences in peak forces, KO filopodia released adhesion, or relaxed force, on the substrate, allowing the beads to return to their original position more quickly. In the eight $\mathrm{KO}$ cases that could be analyzed, seven of the beads had returned to their original location within $5 \mathrm{~min}$. In contrast, in the $10 \mathrm{WT}$ cases that could be analyzed, only one bead had returned to its original position within $5 \mathrm{~min}$.
If filopodia contractions develop less force over shorter time periods, then the amount of momentum necessary for pulling the growth cone forward might be reduced. Reduction in the ability of filopodial interactions to pull the growth cone forward would be expected to be more pronounced when cells are grown in more adhesive substrates. Therefore, to test for the effects of adhesion on outgrowth rates, we plated explants on glass coverslips that were coated with a mixture of laminin and polyornithine. Polyornithine and the related molecule poly-L-lysine have been shown to increase cell adhesiveness to glass substrates and can affect the speed of cell migration (Jay et al., 1995). Consistent with increased adhesiveness, growth cones of both WT and KO cones showed increased numbers of trailing traction fibers compared with growth on laminin alone (Fig. 6, insets). Whereas WT neurons grew at approximately the same rate on the laminin/ polyornithine substrate mixture $(0.39 \pm 0.08 \mu \mathrm{m} / \mathrm{min} ; n=3)$ as on laminin substrates (Tullio et al., 2001), the KO cones grew more slowly $(0.23 \pm 0.04 \mu \mathrm{m} / \mathrm{min} ; n=3$ ) (Fig. 6$)$. Thus the $\mathrm{KO}$ rate of outgrowth on laminin/polyornithine was $59 \%$ slower than WT. This is compared with $\sim 32 \%$ slower on laminin alone (Tullio et al., 2001). This indicates that the KO cones cannot advance as efficiently as WT cones on more adhesive substrates. This supports the idea that decreases in traction force observed in $\mathrm{KO}$ cones can influence forward advancement. In addition, the elongated and narrow shape of the $\mathrm{KO}$ cones grown on the more adhesive substrates suggests that the cones may be compromised in their ability to retract their rear edge. This is consistent with the role of myosin II in Dictyostelium amoebas (Jay et al., 1995).

\section{Myosin IIA may contribute to filopodia traction force}

Because filopodial-based traction forces are still observed in $\mathrm{KO}$ growth cones, additional myosins must contribute to traction force in growth cones. One possibility is that myosin IIA is responsible for traction force. Although myosin IIA appears to be expressed at lower levels than myosin IIB in neurons, it is possible that it may be redistributed or upregulated in cells from the $\mathrm{KO}$ animals. To test for either redistribution or changes in the relative level of expression, we stained WT and $\mathrm{KO}$ growth cones with antibodies to myosin IIA. Some cones also were stained with rhodamine phalloidin to detect the F-actin distribution (Fig. 6), whereas other cones were stained with a volume marker (FITC). In $\mathrm{KO}$ cones the myosin IIA had a distribution similar to that observed in WT cones. In both cone types it was concentrated in the central region of the cone but sometimes also was seen to associate with filopodia. We also analyzed the peak intensity of the myosin IIA staining by using the FITC staining to obtain a ratio. For WT cones the ratio of myosin IIA to FITC was 0.78 , whereas for $\mathrm{KO}$ cones the ratio was 0.75 ( $n=6$ cones each). Thus the staining intensity was the same. From these observations and measurements no detectable redistribution or upregulation of myosin IIA was observed in growth cones.

\section{DISCUSSION}

The absence of myosin IIB in neurons leads to obvious and persistent changes in growth cone motility. Myosin IIB is required for maintaining normal growth cone shape, polarization, size, and actin organization. It is also required for normal rates of shape change and traction force.

\section{Alterations in shape dynamics}

$\mathrm{KO}$ growth cones were small and poorly polarized and changed shape abnormally. Myosin IIB-dependent contraction of cortical 

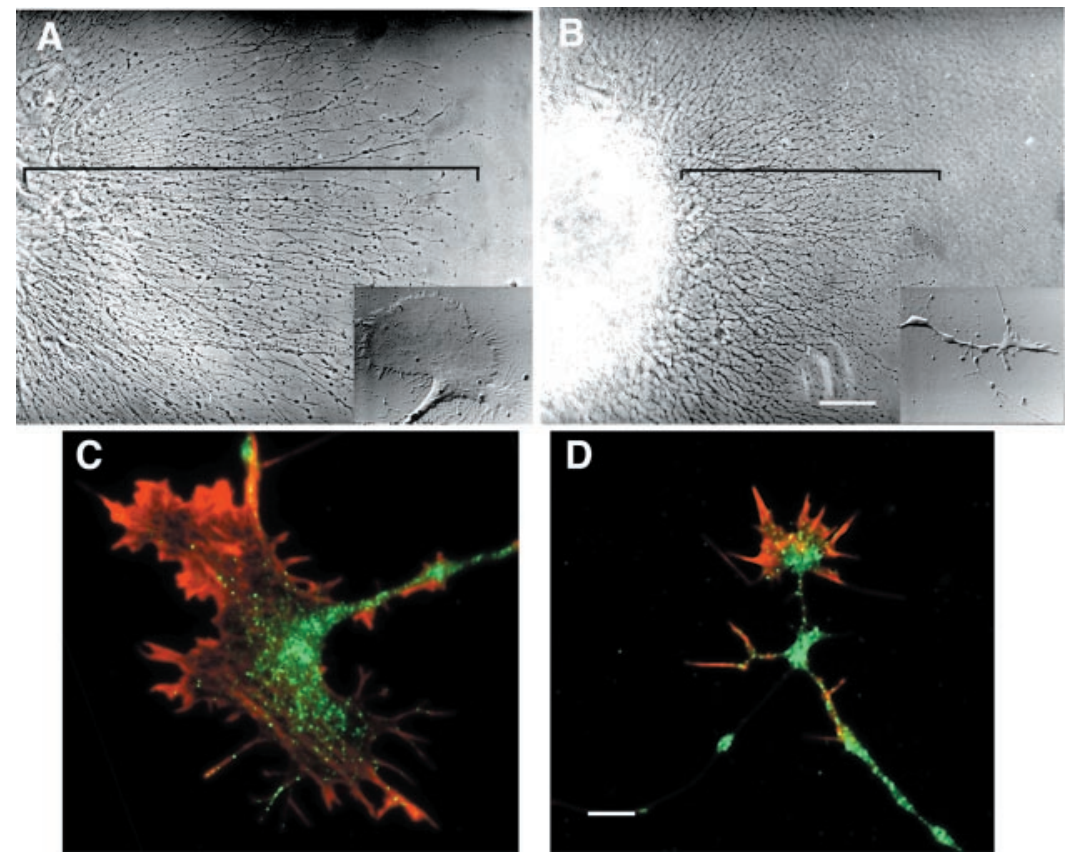

Figure 6. WT $(A)$ and myosin IIB KO $(B)$ radial outgrowth from explants on a relatively adhesive substrate (laminin plus $0.1 \mathrm{mg} / \mathrm{ml}$ polyornithine). The $\mathrm{KO}$ outgrowth is slow compared with WT. Insets, Comparison of growth cone morphology. The cone from the WT animals has a spread morphology. Retraction fibers can be seen at the rear of the cone and along the neurite. Most retraction fibers lie approximately parallel to the neurite axis. In contrast, the cone from the myosin IIB $\mathrm{KO}$ animal is narrow. It has a much smaller area in contact with the adhesive substrate. Retraction fibers are seen approximately perpendicular to the neurite axis. $C, D$, Localization and expression levels of myosin IIA ( green) appear relatively normal in growth cones from myosin IIB KO animals. $C$, Immunofluorescent localization of myosin IIA (green) and F-actin (red) in a WT cone. D, Localization of myosin IIA (green) and actin (red) in a myosin IIB KO cone. In both cones myosin IIA staining is brightest in the central region of the cone. Punctate staining also can be seen more peripherally along actin bundles, which include bundles in filopodia. Quantitative analysis of myosin IIA staining brightness did not reveal any significant differences between WT and KO cones. Scale bars: $B, 114 \mu \mathrm{m} ; D, 4 \mu \mathrm{m}$. actin may increase the stiffness of the cell cortex, inhibiting new protrusive events. In the absence of myosin IIB the softer cell cortex allows for more frequent, less stable protrusive events around the entire perimeter of the cone. The result is a lesspolarized irregular shape and reduced persistence of protrusion in the direction of advance. Thus the results are consistent with the hypothesis that myosin IIB contributes to cortical tension.

\section{Abnormal actin organization}

Myosin IIB KO growth cones have abnormal actin organization. The absence of actin bundles in the marginal zone (palm) is consistent with the model for myosin II-driven retrograde flow and traction force that has been proposed for fish keratocytes (Svitkina et al., 1997). Although growth cones have much more complex morphologies, this model has been used to explain myosin II-dependent retrograde flow and movements of cones (Heidemann and Buxbaum, 1998). Normally, myosin IIB probably drives the retrograde flow and bundling of actin filaments in the marginal zone. Presumably, in the absence of myosin IIB, myosin IIA partially takes over this function but is unable to bundle actin consistently. This also suggests that retrograde flow may be abnormal.

The actin bundles of the marginal zone also may maintain the spread morphology of the cone. In fish keratocytes myosin II contracts actin bundles that arc across the dorsal surface (Burton et al., 1999). Internal shortening of curved actin bundles straightens those attached to a stiff substratum at their ends. The bundles press down on the cytoplasm, squeezing it out toward the periphery and promoting a spread morphology. Also, the contraction causes the cell (cone) to flatten. In the absence of myosin IIB or a rigid substrate, the growth cones would be smaller and thicker, as we have observed.

It has been proposed that myosin II contributes to the spreading between filopodia (Cramer and Mitchison, 1995). KO growth cones are smaller and have reduced spreading between filopodia. Thus, myosin IIB may contribute to this type of spreading via a contractile mechanism similar to that responsible for traction force.

\section{Traction force and filopodial contraction}

Traction force is necessary for growth cone advancement (Lamoureux et al., 1989; Heidemann et al., 1990). Both filopodia and lamellipodia probably contribute to traction force via myosindriven mechanisms (Heidemann et al., 1990; Lin et al., 1996; Svitkina et al., 1997). Although previous studies indicated that myosin contributes to outgrowth and growth cone motility (Lin et al., 1996; Ruchhoeft and Harris, 1997; Wylie et al., 1998), a specific role in traction force had not been tested. We have now tested the contribution of myosin IIB to traction force that is exerted by filopodia by measuring the force in the presence and absence of myosin IIB. A previous estimate of filopodial traction force suggested a range of 50-90 $\mu \mathrm{dyn} /$ filopodium (Heidemann et al., 1990). Our direct measurements (97 $\mu$ dyn/filopodium) in WT neurons are consistent with that estimate. Furthermore, we observed filopodial shortening during the generation of traction force. This is consistent with a contractile mechanism involving myosin II mini-filaments interacting with appropriately oriented actin filaments (Svitkina et al., 1989; Lewis and Bridgman, 1992; Verkhovsky et al., 1995). Filopodial contractions have been observed rarely in cones on rigid glass substrates. Presumably, this is because the contraction is isometric unless the filopodium releases its adhesion to the substrate. Tension transmitted to structures further back in the cone (i.e., lamellipodia between the base of filopodia) will cause them to move forward. The lack of obvious filopodial contraction has led to the assumption that contraction contributes little to traction force. Our results indicate that contractions of filopodia contribute substantially to traction force. Although we limited our comparisons to filopodia, lamellipodia also drive bead movements and thus contribute to traction force. The reduced lamellipodia area in the myosin IIB $\mathrm{KO}$ growth cones, combined with the decrease in cone size, suggests that the traction force reduction per cone is greater than the $30 \%$ decrease seen for filopodia. The mechanism of myosin II-driven traction force in lamellipodia is likely to be similar to that in fish keratocytes (Svitkina et al., 1997).

Although reductions in traction force are likely to result di- 
rectly from the absence of myosin IIB-dependent mechanical force generation, it is also possible that the reduced ability to transfer mechanical energy to the substrate contributes. Myosin II-dependent tension has been implicated in maintaining focal adhesions in fibroblasts (Sastry and Burridge, 2000). Neuronal precursor cells in the myosin IIB KO mice CNS exhibit a disruption in organization, suggesting reduced adhesion (Tullio et al., 2001). Inhibition of myosin IIA in neuroblastoma cells by using antisense oligonucleotides causes the rearrangement of focal contacts and cell detachment (Wylie and Chantler, 2001). However, neuroblastoma cells and primary neurons may react differently to myosin II activity inhibition. Growth cones of primary neurons do not form fully developed focal contacts (Renaudin et al., 1999), and, unlike neuroblastoma cells, the levels of myosin IIA appears to be less than those of myosin IIB (Rochlin et al., 1995). Although we have not tested for reduced adhesion directly, myosin IIB KO cones firmly adhered to laminin or laminin/polyornithine substrates. Furthermore, the decrease in traction force that is associated with filopodia of the KO was less than anticipated and was variable, sometimes reaching levels comparable with WT filopodia. If reduced adhesion caused the decrease in traction force, then myosin IIB would need to regulate adhesion dynamically. It seems more likely that the difference in traction force observed in the myosin IIB KO growth cones results directly from decreases in the levels of mechanical force.

\section{Cooperation between myosins to regulate growth cone motility}

The specific and complete ablation of myosin IIB indicates that it works in cooperation with other myosins to give the full range of motile behavior observed in growth cones. Generation of traction force requires force-producing mechanoenzymes; thus other myosins must contribute to this function. We propose that myosin IIA produces the majority of the traction force observed in the myosin IIB KO growth cones. Although myosin IIA does not appear to be upregulated in KO neurons, myosin IIA has three times greater ATPase and filament sliding speeds compared with myosin IIB (Kelley et al., 1996). Myosin IIA is the only myosin known to be present in growth cones of the KO that could cause filopodial shortening via contractile schemes that use bipolar filaments. Consistent with this possibility, filopodia of the KO growth cones sometimes stained for myosin IIA. Thus myosin IIA could substitute for myosin IIB as the force-producing mechanoenzyme in the smaller growth cones that are present in the KO. However, it seems unlikely that myosin IIA is normally essential for this function. Mutations in the myosin II heavy chain A produce congenital defects that result in thrombocytopenia, giant platelets, and leukocyte inclusions but no obvious neurologic defects (Kelley et al., 2000). We cannot rule out the possibility that other myosins enriched in growth cones, such as myosin Ia, myosin Va, and myosin VI, also play a role in motility (Lewis and Bridgman, 1996; Wang et al., 1996; Evans et al., 1997; Suter et al., 2000).

Occasionally, filopodia-associated beads moved independently of detectable filopodia shortening. Bead movement in these cases could result from forces exerted via a noncontractile-driven retrograde flow mechanism (Lin et al., 1996). Alternatively, the moving beads are proximal to the points of adhesion, and polymerization-dependent protrusion continues more distally.

If retrograde flow and traction force are driven by myosin II-dependent contractions (Svitkina et al., 1997), then the coupling of the force generation system to receptors for substrate adhesion molecules needs further investigation (Suter et al., 1998; Suter and Forscher, 2000). The rate of retrograde flow is proportional to the rate of growth cone advancement (Lin and Forscher, 1995). This suggests that a molecular clutch mechanism slows the retrograde flow rate as force is transferred to the substrate. Suter et al. (1998) recently provided the first evidence for transducing retrograde flow into growth cone movement via a variable linkage between a cell surface receptor and the actin cytoskeleton. This observation, combined with those on myosin II-dependent adhesion (Wei and Adelstein, 2000; Wylie and Chantler, 2001) and the dependence of focal contacts on sustained levels of actin-myosindependent tension in cells (Sastry and Burridge, 2000), suggests that myosin IIA and IIB might act together in shared but distinct roles to drive motility in growth cones. Sustained cytoplasmic tension that is driven by myosin IIA may maintain or organize proteins in cell substrate contacts, whereas the more abundant myosin IIB may provide the force that is required for traction. In the absence of myosin IIB, myosin IIA is required to serve both functions with reduced efficiency, and outgrowth is slowed. Thus myosin IIB is required for normal grow th cone motility and nerve outgrowth rates.

\section{REFERENCES}

Bentley D, Toroian-Raymond A (1986) A disoriented pathfinding by pioneer neuron growth cones deprived of filopodia by cytochalasin treatment. Nature 323:712-715.

Burton K, Park JH, Taylor DL (1999) Keratocytes generate traction force in two phases. Mol Biol Cell 10:3745-3769.

Chien CB, Rosenthal DE, Harris WA, Holt CE (1993) Navigational errors made by growth cones without filopodia in the embryonic $\mathrm{Xe}$ nopus brain. Neuron 11:237-251.

Cramer LP, Mitchison TJ (1995) Myosin is involved in postmitotic cell spreading. J Cell Biol 131:179-189.

Dembo M, Oliver T, Ishihara A, Jacobson K (1996) Imaging the traction stresses exerted by locomoting cells with the elastic substrate method. Biophys J 70:2008-2022.

Evans LL, Hammer J, Bridgman PC (1997) Subcellular localization of myosin $\mathrm{V}$ in nerve growth cones and outgrowth from dilute-lethal neurons. J Cell Sci 110:439-449.

Heidemann SR, Buxbaum RE (1998) Cell crawling: first the motor, now the transmission. J Cell Biol 141:1-4.

Heidemann SR, Lamoureux P, Buxbaum RE (1990) Growth cone behavior and production of traction force. J Cell Biol 111:1949-1957.

Itoh K, Adelstein RS (1995) Neuronal cell expression of inserted isoforms of vertebrate nonmuscle myosin heavy chain II-B. J Biol Chem 270:14533-14540.

Jay PY, Pham PA, Wong SA, Elson EL (1995) A mechanical function of myosin II in cell motility. J Cell Sci 108:387-393.

Kater SB, Rehder V (1995) The sensory-motor role of growth cone filopodia. Curr Opin Neurobiol 5:68-74.

Kelley CA, Sellers JR, Gard DL, Bui D, Adelstein RS, Baines IC (1996) Xenopus nonmuscle myosin heavy chain isoforms have different subcellular localizations and enzymatic activities. J Cell Biol 134:675-687.

Kelley MJ, Jawlen W, Ortel TL, Korczak JF (2000) Mutation of MYH9, encoding non-muscle myosin heavy chain A, in May-Hegglin anomaly. Nat Genet 26:106-108.

Lamoureux P, Buxbaum RE, Heidemann SR (1989) Direct evidence that growth cones pull. Nature 340:159-162.

Lee J, Leonard M, Oliver TN, Ishihara A, Jacobson K (1994) Traction forces generated by locomoting keratocytes. J Cell Biol 127:1957-1964.

Lewis AK, Bridgman PC (1992) Nerve growth cone lamellipodia contain two populations of actin filaments that differ in organization and polarity. J Cell Biol 119:1219-1243.

Lewis AK, Bridgman PC (1996) Mammalian myosin $1 \alpha$ is concentrated near the plasma membrane in nerve growth cones. Cell Motil Cytoskeleton 33:130-150.

Lin CH, Forscher P (1995) Growth cone advance is inversely proportional to retrograde F-actin flow. Neuron 14:763-771.

Lin CH, Espreafico EM, Mooseker MS, Forscher P (1996) Myosin drives retrograde F-actin flow in neuronal growth cones. Neuron 16:769-782.

Mallavarapu A, Mitchison T (1999). Regulated actin cytoskeleton assembly at filopodium tips controls their extension and retraction. J Cell Biol 146:1097-1106.

Oliver T, Dembo M, Jacobson K (1995) Traction forces in locomoting cells. Cell Motil Cytoskeleton 31:225-240.

Pelham RJ, Wang Y-L (1997) Cell locomotion and focal adhesions are 
regulated by substrate flexibility. Proc Natl Acad Sci USA 94:13661-13665.

Pelham RJ, Wang Y-L (1999) High resolution detection of mechanical forces exerted by locomoting fibroblasts on the substrate. Mol Biol Cell 10:935-945.

Renaudin A, Lehmann M, Girault JA, McKerracher L (1999) Organization of point contacts in neuronal growth cones. J Neurosci Res 55:458-471.

Rochlin MW, Itoh K, Adelstein RS, Bridgman PC (1995) Localization of myosin II A and B isoforms in cultured neurons. J Cell Sci 108:3661-3670.

Ruchhoeft ML, Harris WA (1997) Myosin functions in Xenopus retinal ganglion cell growth cone motility in vivo. J Neurobiol 32:567-578.

Sastry SK, Burridge K (2000) Focal adhesions: a nexus for intracellular signaling and cytoskeletal dynamics. Exp Cell Res 261:25-36.

Suter DM, Forscher P (2000) Substrate-cytoskeletal coupling as a mechanism for the regulation of growth cone motility and guidance. J Neurobiol 44:97-113.

Suter DM, Errante LD, Belotserkovsky V, Forscher P (1998) The Ig superfamily cell adhesion molecule, apCAM, mediates growth cone steering by substrate-cytoskeletal coupling. J Cell Biol 141:227-240.

Suter DM, Espindola FS, Lin C-H, Forscher P, Mooseker MS (2000) Localization of unconventional myosins $\mathrm{V}$ and VI in neuronal growth cones. J Neurobiol 42:370-382.

Svitkina TM, Surguchova IG, Verkhovsky AB, Gelfand VI, Moeremans M, Mey J-D (1989) Direct visualization of bipolar myosin filaments in stress fibers of cultured fibroblasts. Cell Motil Cytoskeleton 12:150-156.

Svitkina TM, Verkhovsky AB, Mcquade KM, Borisy GG (1997) Analysis of the actin-myosin II system in fish epidermal keratocytes mechanism of cell body translocation. J Cell Biol 139:397-415.

Tullio AN, Accili D, Ferrans VJ, Yu Z-X, Takeda K, Grinberg A,
Westphal H, Preston YA, Adelstein RS (1997) Nonmuscle myosin II-B is required for normal development of the mouse heart. Proc Natl Acad Sci USA 94:12407-12412.

Tullio AN, Bridgman PC, Tresser NJ, Chan, C-C, Conti MA, Adelstein RS, Hara Y (2001) Structural abnormalities develop in the brain following ablation of the gene encoding nonmuscle myosin II-B heavy chain. J Comp Neurol 433:62-74.

Uren D, Hwang HK, Hara Y, Takeda K, Kawamoto S, Tullio AN, Yu ZX, Ferrans VJ, Tresser N, Grinberg A, Preston YA, Adelstein RS (2000) Gene dosage affects the cardiac and brain phenotype in nonmuscle myosin II-B-depleted mice. J Clin Invest 105:663-671.

Verkhovsky AB, Svitkina TM, Borisy GG (1995) Myosin II filament assemblies in the active lamella of fibroblasts: their morphogenesis and role in the formation of actin filament bundles. J Cell Biol 131:989-1002.

Wakatsuki T, Kolodney MS, Zahalak GI, Elson EL (2000) Cell mechanics studied by a reconstituted model tissue. Biophys J 79:2353-2368.

Wang F-S, Wolenski JS, Cheney RE, Mooseker MS, Jay DG (1996) Function of myosin- $\mathrm{V}$ in filopodia extension of neuronal growth cones. Science 273:660-663.

Wei Q, Adelstein RS (2000) Conditional expression of truncated fragment of nonmuscle myosin II-A alters cell shape but not cytokinesis in HeLa cells. Mol Biol Cell 11:3617-3627.

Wylie SR, Chantler PD (2001) Separate but linked functions of conventional myosins modulate adhesion and neurite outgrowth. Nat Cell Biol 3:88-92.

Wylie SR, Wu P-J, Patel H, Chantler PD (1998) A conventional myosin motor drives neurite outgrowth. Proc Natl Acad Sci USA 95:12967-12972.

Zheng JQ, Wan J-J, Poo M-M (1996) Essential role of filopodia in chemotropic turning of nerve growth cone induced by a glutamate gradient. J Neurosci 16:1140-1149. 